\title{
Ciprofloxacin prophylaxis in high risk neutropenic patients: effects on outcomes, antimicrobial therapy and resistance
}

\author{
Marcia Garnica ${ }^{1}$, Simone A Nouér ${ }^{1}$, Flávia LPC Pellegrino ${ }^{1,2}$, Beatriz M Moreira ${ }^{2}$, Angelo Maiolino ${ }^{1}$ and Marcio Nucci ${ }^{* *}$
}

\begin{abstract}
Background: The use of quinolone prophylaxis in high-risk neutropenic patients is considered standard of care but the development of resistance is a concern. Previous studies have focused mainly on quinolone resistance among patients receiving prophylaxis, with very few data reporting its impact on the hospital microbial epidemiology.

Methods: We analyzed a cohort of 329 episodes of chemotherapy-induced neutropenia in adults, and compared two periods: 2005 (period 1, no prophylaxis, $\mathrm{n}=110$ ) and 2006-2008 (period 2, ciprofloxacin prophylaxis, $\mathrm{n}=219$ ). Outcomes analyzed were the frequency of febrile neutropenia, bacteremia, duration of antibiotic therapy and hospitalization, and antimicrobial resistance to ciprofloxacin and extended-spectrum beta-lactamase [ESBL] production. We analyzed resistance rates (by patients-day) in the cohort, as well as in other patients (neutropenic and non-neutropenic, 11,975 patients-day) admitted to the hematology unit in the same period, taking into consideration the general resistance patterns in the hospital.

Results: Quinolone prophylaxis (period 2) resulted in fewer episodes of febrile neutropenia (159/219 [73\%] vs. 102/110 [93\%], Chi-square 18.09, $p=0.00002$ ), and bacteremia (49/219 [22] vs. 36/110 [33\%], Chi-square 4.10, $p=0.04)$, shorter duration of antibiotic therapy $(p=0.0002)$ and hospitalization $(p=0.002)$, but more frequent use of carbapenems (79/219 [36\%] vs. 15/110 [14\%], Chi-square 18.06, $p=0.0002)$. In addition, period 2 was associated with higher rates of quinolone resistance (6.77 vs. 3.02 per 1,000 patients-day, $p=0.03$ ). The rate of ESBL-producing enterobacteria in the two periods was slightly higher in patients receiving quinolone prophylaxis (1.27 vs. 0.38 per 1,000 patients-day, $p=0.26)$ as well as in the hematology unit overall (1.59 vs. 0.53 per 1,000 patients-day, $p=0.08$ ), but remained stable in the whole hospital ( $0.53 \mathrm{vs}$. 0.56 per 1,000 patients-day, $p=0.74)$.
\end{abstract}

Conclusions: Ciprofloxacin prophylaxis was beneficial in high risk neutropenic patients, but important modifications in the prescription of carbapenems and on antimicrobial resistance patterns of isolates were observed. The importance of hospital or ward ecology must be taken into account when deciding for quinolone prophylaxis in high-risk neutropenic patients.

Keywords: Neutropenia, Prophylaxis, Quinolone, Febrile neutropenia, Resistance

\footnotetext{
*Correspondence: mnucci@hucff.ufrj.br

${ }^{1}$ Hospital Universitário Clementino Fraga Filho, Universidade Federal do Rio de Janeiro, Rua Professor Rodolpho Paulo Rocco 255, Cidade Universitária, Rio de Janeiro 21941-913, Brazil

Full list of author information is available at the end of the article
} 


\section{Background}

The use of quinolone prophylaxis in neutropenic cancer patients has been associated with a reduction in the incidence of bacterial infections and mortality [1-4]. However, an increase in quinolone resistance [5-7], as well as resistance to other classes of antimicrobial agents, such as extended-spectrum beta-lactamase (ESBL) production among enterobacteria has been reported [8-12]. The potential increase in resistance with the use of quinolones is relevant in the neutropenic setting, because the high incidence of infection caused by ESBL-producing bacteria limits the options for empirical treatment of febrile neutropenia. Furthermore, in a setting of a high prevalence of Gram-negative resistance, the use of inappropriate antibiotic therapy results in significant excess mortality [13]. Studies reporting antimicrobial resistance among neutropenic patients receiving quinolone prophylaxis have focused mostly on the emergence of quinolone resistance among patients receiving prophylaxis, with very few data reporting its impact on the hospital microbial epidemiology $[5,14]$.

In Brazil the rates of Gram-negative bacteremia in neutropenic patients are high [15], rendering quinolone prophylaxis an attractive approach. However, the rates of resistance among Gram-negative bacilli are high in the region [16]. Therefore, an analysis of the potential benefits of quinolone prophylaxis must be carefully weighed against the risks of resistance. In the present study we evaluated the impact of quinolone prophylaxis given during neutropenia on different outcomes, with special attention to the rates of resistance among patients on prophylaxis, as well as in patients admitted to the hematology unit in the same period, taking into consideration the general resistance patterns in the hospital.

\section{Methods}

We conducted an observational study at Hospital Universitário Clementino Fraga Filho, Federal University of Rio de Janeiro, Brazil. This is a tertiary care hospital with $\sim 400$ beds, including a hematology and hematopoietic cell transplant (HCT) unit with 8 single-bed rooms with high efficiency particulate air (HEPA) filter and positive pressure, and five double-bed rooms without HEPA filter. The study was approved by the institution's Ethical Committee ("Comitê de Ética em Pesquisa do Hospital Universitário Clementino Fraga Filho”).

Until 2006, no antibacterial prophylaxis had been given to neutropenic patients. Since then, all patients with hematological malignancies with an expected duration of neutropenia $>7$ days received prophylaxis with ciprofloxacin (500 mg orally twice a day, switched to $200 \mathrm{mg}$ intravenously twice a day if the patient developed severe mucositis or intolerance to the oral formulation). Prophylaxis was started concomitantly with the induction chemotherapy or the conditioning regimen ( $\mathrm{HCT}$ ), and was maintained until bone marrow recovery or fever. In case of fever, blood cultures were obtained, and the patients were immediately started on intravenous cefepime, unless a previous episode of febrile neutropenia documented a cefepime-resistant Gram-negative organism. In this case, a carbapenem (imipenem or meropenem) was started. Blood cultures were repeated in case of persistent or recurrent fever, or as clinically indicated. Modifications in the empirical antibiotic regimen were performed according to the results of cultures and the clinical course of the patient.

For the purpose of this analysis, we selected all patients admitted between 2005 and 2008 who fulfilled the following criteria: a) receipt of chemotherapy with expected duration of neutropenia $>7$ days; and b) no fever or documentation of infection on the first day of chemotherapy. We then compared patients who received prophylaxis with ciprofloxacin (2006-2008, period 2, ciprofloxacin group) with patients who did not receive any antibiotic prophylaxis (2005, period 1, control group). Patients could be included more than once provided that more than 30 days had elapsed between two episodes of neutropenia. The groups were compared regarding demographic characteristics (age, gender), underlying disease, type of treatment (induction or intensification for acute leukemia), type of HCT (autologous or allogeneic), Karnofsky's performance status, presence and severity of mucositis (Common Toxicity Criteria of National Cancer Institute World Health Organization criteria), and duration of neutropenia. We analyzed the following clinical outcomes: occurrence of fever, duration of empirical antibiotic therapy, bacteremia, bacteremia due to ciprofloxacin-resistant organism, bacteremia due to ESBL-producing enterobacteria, duration of hospitalization and antimicrobial therapy, use of carbapenem and glycopeptide, and death during the episode of neutropenia. All data were collected prospectively, as part of a large database of febrile neutropenia.

Neutropenia was defined as an absolute neutrophil count $(\mathrm{ANC})<500 / \mathrm{mm}^{3}$, and severe neutropenia as an ANC $<100 / \mathrm{mm}^{3}$. Bone marrow recovery was defined as at least two consecutive ANCs $>500 / \mathrm{mm}^{3}$, obtained on two separate days. Fever was defined as an axillary temperature $>38^{\circ} \mathrm{C}$. The febrile episodes were classified as fever of unknown origin (FUO), bacteremia, microbiologically documented infection without bacteremia, or clinically documented infection, as previously defined [17].

Polymicrobial bacteremia was defined if more than one pathogen was isolated from one or more blood cultures taken on the same day during the febrile episode. Blood cultures were processed with the BacT/ALERT system (Organon Teknika, USA). Bacterial identification and antimicrobial susceptibility tests were performed 
using the Vitek automated system (Bio-Merieux, Inc., France). No changes in microbiological procedures took place during the study period (2005-2008).

All bloodstream isolates from this cohort that had been stored $\left(-80^{\circ} \mathrm{C}\right)$ were reprocessed to evaluate susceptibility. The following tests were performed: antimicrobial susceptibility test by disk-diffusion [18], minimal inhibitory concentration (MIC) of ciprofloxacin by E-test (Probac do Brasil), and determination of ESBL production by enterobacteria, with a double-diffusion test (using ceftazidime, cefepime, cefotaxime and aztreonam containing disks as substrates, and amoxicillin-clavulanate containing disks as inhibitor) [19]. If the isolate was not available for these additional procedures, data were obtained from the records of the microbiology laboratory.

In order to rule out the possibility that horizontal transmission of isolates occurred during the study period, molecular typing of Gram-negative isolates was performed using polymerase chain reaction (PCR)-fingerprinting with the following primers: ERIC-2 for Escherichia coli, Klebsiella spp., Enterobacter spp., and Citrobacter freundii [20,21], and 272 for Serratia marcescens and Proteus mirabilis isolates [22]. Banding patterns were interpreted by visual inspection and with GelCompar II (version 4.01), using the Dice index and the unweighted pair group method with arithmetic averages (UPGMA).

In addition to the analysis of resistance among patients in the cohort, we looked at the rates of resistance of bloodstream isolates obtained from patients of the unit who were not in the cohort, as well as the rates in other units of the hospital in the same 4-year period. Patients not in the cohort comprised 11,975 patients-day and included both neutropenic and non-neutropenic patients. We looked specifically at the rates of ciprofloxacin resistance and ESBL production. Resistance rates were reported per 1,000 patients.day.

All statistical analyses were performed using the SPSS for Windows software (version 11.0.1, SPSS, Inc., USA). The Chi-square test was used to compare proportions, and Mann-Whitney test to compare continuous variables; $p$ values $<0.05$ were considered statistically significant.

\section{Results}

We analyzed 220 patients (141 in the ciprofloxacin and 79 in the control group) who developed 329 episodes of neutropenia, 219 receiving ciprofloxacin prophylaxis (ciprofloxacin group) and 110 without quinolone prophylaxis (control group). Patients in the ciprofloxacin and control group had similar ages (mean 40 years, range $14-82$ and 41 years, range $12-66$, respectively, $p=0.56$ ) and gender $(60 \%$ and $65 \%$ of males, respectively, $p=0.28)$. Ciprofloxacin prophylaxis was given for a mean of 11 days (range $1-30, \mathrm{SD} \pm 4.44$ ), and was started before neutropenia in $94 \%$ of episodes, at a mean of 7 days before neutropenia $(8-23, \mathrm{SD} \pm 3.49)$.

Table 1 shows the characteristics and outcomes of the episodes of neutropenia in the two groups. The duration of neutropenia was slightly shorter in the ciprofloxacin group ( 9 vs. 11 days, $p=0.02$ ). In addition, mucositis (at any grade, but not grades 3 or 4 ) was less frequent in ciprofloxacin recipients ( $52 \%$ vs. $70 \%, p=0.003$ ). Febrile episodes were significantly less frequent in the ciprofloxacin group (73 vs. 93\%, $p<0.001$ ), and when present, occurred later in the course of neutropenia (median 4 vs. 2 days after the first day of neutropenia, $p<0.001$ ). While the proportion of episodes classified as FUO and clinically documented infection was similar in the two groups, bacteremia was significantly less frequent in the ciprofloxacin group ( $22 \%$ vs. $33 \%, p=0.04)$. In addition, the mean duration of hospitalization was shorter in the ciprofloxacin group (22 vs. 24 days, $p=0.002$ ), as was the mean duration of antibiotic therapy ( 8 vs. 11 days, $p<0.001$ ) (Additional file 1).

The rates of clinical or microbiological failure to the empirical treatment with cefepime were similar in the ciprofloxacin and control groups (12\% vs. $8 \%$ for clinical failures, $p=0.33$; and $8.5 \%$ and $6 \%$ for microbiological failures, $p=0.14$; respectively). In addition, no differences were observed in the frequency of glycopeptide use between the two groups. However, carbapenems were given more frequently to ciprofloxacin recipients (36\% vs. $14 \%$, $p<0.001)$.

A total of 98 bacterial isolates were recovered from the 85 episodes of bacteremia. In the ciprofloxacin group, there were 19 episodes with documentation of a single Gram-negative, 22 episodes with a single Gram-positive, and 8 episodes of polymicrobial bacteremia, whereas in the control group there were 13 episodes with documentation of a single Gram-negative, 18 episodes of a single Gram-positive isolate, and 5 episodes of polymicrobial bacteremia (Table 1). Table 2 shows the species distribution of bloodstream isolates in both groups. The most frequent Gram-negative bacteria were E. coli and $P$. aeruginosa.

Table 3 shows the resistance rates (per 1,000 patients. day) in the two periods. The rate of quinolone-resistant bacteremia (both Gram-positive and Gram-negative) was significantly higher in patients receiving quinolone prophylaxis (6.77 vs. 3.02 per 1,000 patients.day, $p=0.03$ ). In addition, quinolone-resistant enterobacteria were more frequently isolated in period 2 both in cohort patients and in the hematology unit overall ( 2.12 vs. 0.38 per 1,000 patients.day, $p=0.06$ in cohort patients, and 2.54 vs. 0.53 per 1,000 patients.day in the hematology unit, $p=0.004$ ), but remained stable in the hospital ( 0.76 in period 1 and 0.64 in period $2, p=0.15$ ). The rates of ESBL production among enterobacteria increased slightly both in cohort 
Table 1 Characteristics and outcomes of 329 neutropenic episodes in 220 patients who received or not ciprofloxacin prophylaxis during neutropenia

\begin{tabular}{|c|c|c|c|}
\hline Variable & $\begin{array}{l}\text { Ciprofloxacin } \\
\text { group } N=219\end{array}$ & $\begin{array}{c}\text { Control } \\
\text { group } \\
\mathrm{N}=110\end{array}$ & $\begin{array}{c}p \\
\text { value }\end{array}$ \\
\hline \multicolumn{4}{|l|}{ Underlying disease } \\
\hline Acute myeloid leukemia & $48(22)$ & $22(20)$ & 0.69 \\
\hline Acute lymphoid leukemia & $48(22)$ & $24(22)$ & 0.98 \\
\hline Multiple myeloma & $56(26)$ & $31(28)$ & 0.61 \\
\hline Non-Hodgkin lymphoma & $40(18)$ & $8(7)$ & 0.008 \\
\hline Hodgkin lymphoma & $18(8)$ & $15(14)$ & 0.12 \\
\hline Other* & $9(4)$ & $10(9)$ & - \\
\hline Autologous HCT & $89(41)$ & $53(48)$ & 0.19 \\
\hline Allogeneic HCT & $30(14)$ & $14(13)$ & 0.81 \\
\hline Central venous catheter & $140(64)$ & $73(66)$ & 0.66 \\
\hline Performance status $<50 \%$ & $25(11)$ & $19(17)$ & 0.14 \\
\hline Mucositis, any grade & $114(52)$ & $76(70)$ & $<0.001$ \\
\hline Grade 3 or 4 & $28(13)$ & $16(15)$ & 0.66 \\
\hline $\begin{array}{l}\text { Duration (days) of neutropenia, } \\
\text { mean } \pm \mathrm{SD} \text { (range) }\end{array}$ & $9 \pm 6.3(2-47)$ & $\begin{array}{c}11 \pm 7.9 \\
(2-61)\end{array}$ & 0.02 \\
\hline $\begin{array}{l}\text { Duration (days) of severe } \\
\text { neutropenia, mean } \pm \mathrm{SD} \text { (range) }\end{array}$ & $7 \pm 5.2(1-38)$ & $\begin{array}{l}8 \pm 6.9 \\
(0-40)\end{array}$ & 0.39 \\
\hline Fever & $159(73)$ & $102(93)$ & $<0.001$ \\
\hline Fever of unknown origin & $86(39)$ & $52(47)$ & 0.16 \\
\hline Bacteremia & $49(22)$ & $36(33)$ & 0.04 \\
\hline $\begin{array}{l}\text { due to a single } \\
\text { Gram-negative }\end{array}$ & $19(9)$ & $13(12)$ & 0.36 \\
\hline $\begin{array}{l}\text { due to a single } \\
\text { Gram-positive }\end{array}$ & $22(10)$ & $18(16)$ & 0.10 \\
\hline Polymicrobial & $8(4)$ & $5(4.5)$ & 0.77 \\
\hline $\begin{array}{l}\text { Microbiologically } \\
\text { documented without bacteremia }\end{array}$ & $3(1)$ & $1(1)$ & 1.00 \\
\hline Clinically documented & $21(10)$ & $12(11)$ & 0.71 \\
\hline $\begin{array}{l}\text { Duration of hospitalization (days), } \\
\text { mean } \pm \mathrm{SD} \text { (range) }\end{array}$ & $\begin{array}{c}22 \pm 13.9(4- \\
97)\end{array}$ & $\begin{array}{l}24 \pm 10.4 \\
(5-57)\end{array}$ & 0.002 \\
\hline $\begin{array}{l}\text { Duration of antimicrobial } \\
\text { treatment (days), mean } \pm S D \\
\text { (range) }\end{array}$ & $8 \pm 7.6(0-40)$ & $\begin{array}{c}11 \pm 7.0(0 \\
-33)\end{array}$ & $<0.001$ \\
\hline Receipt of carbapenem** & $79(36)$ & $15(14)$ & $<0.001$ \\
\hline Receipt of glycopeptide & $26(24)$ & $14(13)$ & 0.82 \\
\hline Death & $20(9)$ & $12(11)$ & 0.61 \\
\hline
\end{tabular}

Data are number (\%) of episodes, unless otherwise indicated.

* Other underlying diseases: Ciprofloxacin group: aplastic anemia (5 episodes), and chronic myeloid leukemia and germ cell tumor (1 each); control group: chronic myeloid leukemia (9), and aplastic anemia (1).

** As empirical therapy in 43 episodes in the ciprofloxacin group and 1 in the control group; as a modification of the empirical regimen in 14 episodes in the ciprofloxacin group and in 36 episodes in the control group.

patients (0.38 in period vs.1.27 in period $2, p=0.26)$ and in the hematology unit ( 0.52 in period 1 vs. 1.59 in period $2, p=0.08)$, and remained stable in the hospital (0.56 in
Table 2 Species distribution of 98 bacterial bloodstream isolates recovered from patients who received ciprofloxacin prophylaxis or not during neutropenia

\begin{tabular}{lcc}
\hline Microorganism & Ciprofloxacin group & Control group \\
\hline $\begin{array}{l}\text { Gram-positive } \\
\text { Coagulase-negative }\end{array}$ & $\mathrm{N}=28$ & $\mathrm{~N}=24$ \\
staphylococci & 14 & 16 \\
Staphylococcus aureus & 4 & 1 \\
$\begin{array}{l}\text { a-Hemolytic streptococci } \\
\text { Other Gram-positive* }\end{array}$ & 5 & 4 \\
Gram-negative & 5 & 3 \\
Escherichia coli & $\mathrm{N}=29$ & $\mathrm{~N}=17$ \\
Klebsiella pneumoniae & 9 & 5 \\
Other enterobacteria** & 2 & 1 \\
Pseudomonas aeruginosa & 4 & 4 \\
Other non-fermentative & 3 & 4 \\
Gram-negative*** & 11 & 3
\end{tabular}

The total number of isolates exceeds the number of bacteremias because there were 13 episodes of polymicrobial bacteremia.

* Ciprofloxacin group: Enterococcus sp. and Streptococcus pneumoniae (1 each), and 3 Gram-positive rods; Control group: Enterococcus sp. (2) and Bacillus sp. (1). ** Ciprofloxacin group: Enterobacter sp. (3), and Shigella sp. (1); Control group: Citrobacter sp. (2), and Enterobacter sp. and Serratia sp. (1 each).

*** Ciprofloxacin group: 5 Acinetobacter sp., 3 Stenotrophomonas maltophilia, and 3 Burkholderia cepacia; Control group: 1 Acinetobacter sp., 1

Stenotrophomonas maltophilia, and 1 Burkholderia cepacia.

period 1 vs. 0.53 in period 2, $p=0.74$ ). No changes in quinolone resistance among staphylococci were observed in cohort patients.

The genotypic analysis of the isolates from the cohort patients showed a great genetic diversity among isolates recovered from episodes of the cohort, with no similarity between isolates (Figure 1).

\section{Discussion}

In this study we observed that ciprofloxacin prophylaxis in high-risk neutropenic patients was associated with a decrease (and delay) in the occurrence of fever, bacteremia, and duration of antibiotic therapy and hospitalization. On the other hand, an increase in the frequency of quinolone resistance was observed, not only in patients receiving quinolones but in the whole hematology unit. In addition, the incidence of bacteremia due to ESBLproducing enterobacteria increased in the hematology unit, while in the hospital it remained stable.

Quinolones have been used as prophylaxis in neutropenic patients since the early 1990s. While its use has been associated with favorable outcomes, the drawback of resistance has been always a concern. Nevertheless, quinolone prophylaxis is considered standard of care in high risk neutropenic patients admitted to centers in which quinolone resistance is $<30 \%[23,24]$.

The beneficial effects of quinolone prophylaxis observed in the present study were reported in the majority of 
Table 3 Incidence rates (per 1,000 patients-day) of resistant bacteria

\begin{tabular}{|c|c|c|c|}
\hline & $\begin{array}{l}\text { Period } 1 \\
(2005)\end{array}$ & $\begin{array}{c}\text { Period 2 } \\
(2006-2008)\end{array}$ & $\begin{array}{c}p \\
\text { value }\end{array}$ \\
\hline \multicolumn{4}{|c|}{ Cohort patients } \\
\hline $\begin{array}{l}\text { Bacteremia due to Cip-R } \\
\text { organisms* }\end{array}$ & 3.02 & 6.77 & 0.03 \\
\hline Cip-R enterobacteria & 0.38 & 2.12 & 0.06 \\
\hline Cip-R Pseudomonas aeruginosa & 0.38 & 0.63 & 0.71 \\
\hline ESBL production & 0.38 & 1.27 & 0.26 \\
\hline Cip-R Staphylococcus aureus & 0 & 0.63 & 0.26 \\
\hline Cip-R CONS & 2.27 & 2.75 & 0.72 \\
\hline \multicolumn{4}{|c|}{ Non-cohort patients in the hematology unit } \\
\hline Cip-R enterobacteria & 0.53 & 2.54 & 0.004 \\
\hline Cip-R Pseudomonas aeruginosa & 0.70 & 0.32 & 0.39 \\
\hline ESBL production & 0.52 & 1.59 & 0.08 \\
\hline Cip-R Staphylococcus aureus & 0 & 0.16 & 0.52 \\
\hline Cip-R CONS & 2.64 & 2.23 & 0.65 \\
\hline \multicolumn{4}{|c|}{ Hospital } \\
\hline Cip-R enterobacteria & 0.76 & 0.64 & 0.15 \\
\hline Cip-R Pseudomonas aeruginosa & 0.22 & 0.21 & 0.78 \\
\hline ESBL production & 0.56 & 0.53 & 0.74 \\
\hline Cip-R Staphylococcus aureus & 0.33 & 0.23 & 0.06 \\
\hline Cip-R CONS & 0.68 & 0.60 & 0.33 \\
\hline
\end{tabular}

$C i p-R=$ ciprofloxacin-resistant; $E S B L$ = extended spectrum beta-lactamase; CONS = coagulase-negative staphylococci; * Total number of bacteremia due to quinolone-resistant organisms available in the cohort only. randomized clinical trials. In one multicenter trial conducted in Italy, the use of levofloxacin in patients with expected duration of neutropenia $>7$ days resulted in a reduction in episodes of febrile neutropenia and bacteremia [1]. In another trial, the same benefits were observed, but there was an increase in the use of carbapenems [25], as observed in the present study.

While these studies give support to the use of quinolone prophylaxis in a select group of neutropenic patients, some important aspects regarding antimicrobial resistance have not been addressed. For example, the Italian trial showed that the rate of levofloxacin-resistant Gram-negative bloodstream isolates was 4-fold higher in levofloxacin recipients, but data regarding the frequency of resistance to other antimicrobial classes, such as betalactams, were not provided [1]. Likewise, other studies emphasized the emergence of quinolone resistance but provided few data regarding increasing of resistance to other antimicrobials [26-28]. The relationship between quinolone exposure and the emergency of ESBL production among enterobacteria is well documented, and is thought to be a result of the mutant window, a phenomenon in which a quinolone-resistant isolate is more likely to acquire other mechanisms of resistance [9].

The increased use of carbapenem in the second period of our study was mostly as primary antibiotic regimen for the first fever (43 episodes in the ciprofloxacin group vs. 1 in the control group). The increased use of carbapenems in the empirical regimen may have been due to a concern of clinicians when we started to experience an increase
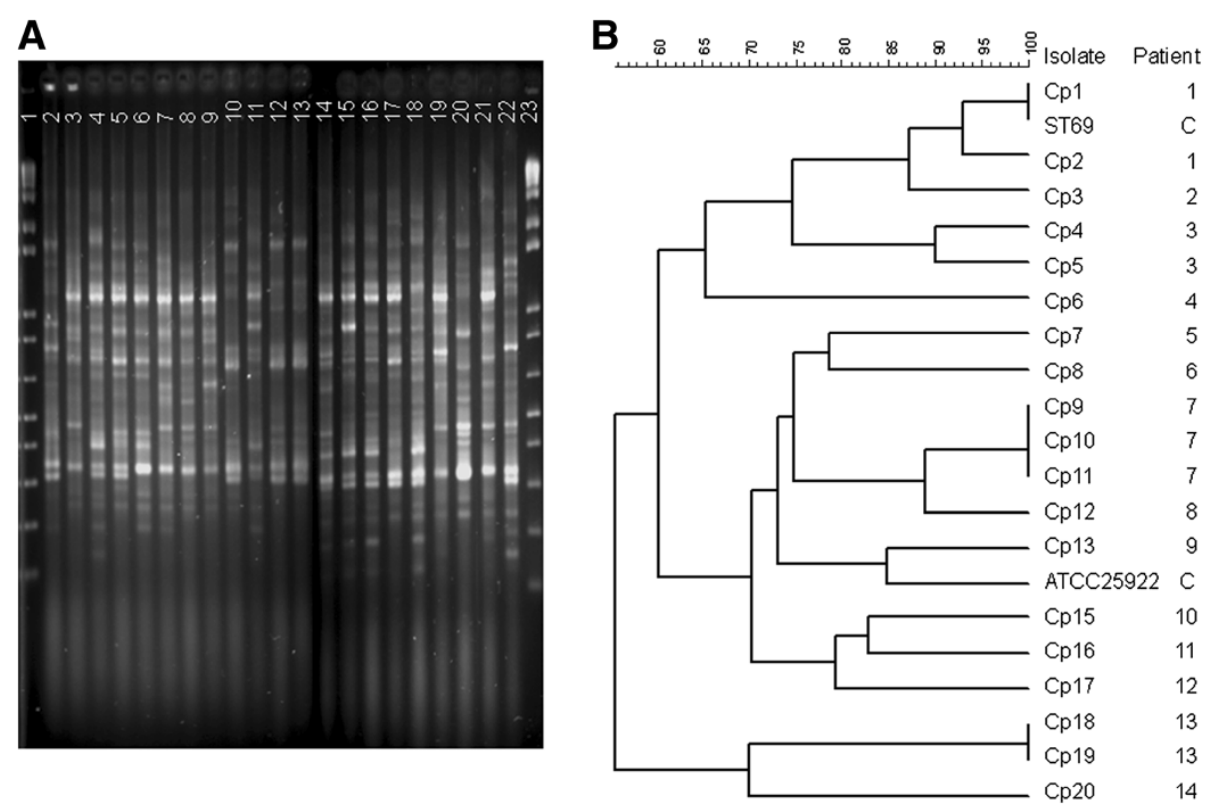

Figure 1 Molecular analysis of Escherichia coli isolates from cohort patients. A. ERIC2-PCR profiles of Escherichia coli isolates. Lanes 1 and 23: 1 Kb plus molecular size marker; Lanes 2-20: clinical isolates; Lanes 21 and 22: control strains ST69 and ATCC25922. B. Dendrogram of ERIC-2 PCR profiles of Escherichia coli isolates. C: control isolates. 
in the rate of bacteremia due to ESBL-producing enterobacteria. Although not significant, there was a slight increase in the recovery of ESBL-producing enterobacteria in period 2 both in the cohort group and in the hematology unit. However, a similar trend was not observed in the hospital in the same period. These data indicate that the increase in ESBL in the hematology unit was not a reflection of an increase in ESBL in the hospital, and suggest a relationship between quinolone use and ESBL. In support of this hypothesis are various studies reporting a close association between quinolone resistance and ESBL $[9,29]$. Furthermore, the lack of genetic similarity among the bloodstream isolates suggests that horizontal transmission was not the main factor responsible for the increase in the incidence of quinoloneresistant and ESBL-producing enterobacteria. Therefore, a careful judgment of the risks and benefits of quinolone resistance should be advanced.

Our study is limited by the fact that we used a historical control group. However, except for time (two different periods) and antibacterial prophylaxis policy (ciprofloxacin or not), no other intervention was carried out that could strongly influence the results. Specifically, no changes in antimicrobial use in the unit or in microbiological procedures occurred in the two periods. Another limitation of our study is that not all bacteria recovered from blood cultures in the period were available for further laboratory analysis.

\section{Conclusion}

In conclusion, ciprofloxacin prophylaxis was beneficial in high risk neutropenic patients, but important modifications in the prescription of carbapenems and on antimicrobial resistance patterns of isolates were observed. The importance of hospital or ward ecology must be taken into account when deciding for quinolone prophylaxis in high-risk neutropenic patients.

\section{Additional file}

Additional file 1: Distribution of duration of neutropenia (A), severe neutropenia (B), hospitalization (C) and antimicrobial treatment (D) in Control and Ciprofloxacin Groups by Box-plots Graphs.

\section{Abbreviations \\ ESBL: Extended-spectrum beta-lactamase; HCT: Hematopoietic cell transplantation; HEPA: High efficiency particulate air; ANC: Absolute neutrophil count; FUO: Fever of unknown origin; MIC: Minimum inhibitory concentration; PCR: Polymerase chain reaction.}

\section{Competing interests}

The authors declare that they have no competing interests.

\section{Authors' contributions}

MG designed the study, collected and analyzed data, and drafted the paper; SAN designed the study, analyzed data, contributed with intellectual input for the discussion and reviewed the final version of the manuscript; FLPCP processed the isolates and approved the final version of the manuscript; BMM contributed with intellectual input for the discussion and reviewed the final version of the manuscript; AM contributed with intellectual input for the discussion and reviewed the final version of the manuscript; MN designed the study, analyzed data, contributed with intellectual input for the discussion, and reviewed and approved the final version of the manuscript. All authors read and approved the final manuscript.

\section{Acknowledgements}

This study was supported in part by Conselho Nacional de Desenvolvimento Científico e Tecnológico, CNPq, Brazil, grants 472154/2008-7 and 301025/ 2008-8.

\section{Author details}

${ }^{1}$ Hospital Universitário Clementino Fraga Filho, Universidade Federal do Rio de Janeiro, Rua Professor Rodolpho Paulo Rocco 255, Cidade Universitária, Rio de Janeiro 21941-913, Brazil. ${ }^{2}$ Instituto de Microbiologia Paulo de Góes, Universidade Federal do Rio de Janeiro, Rio de Janeiro, Brazil.

Received: 22 August 2012 Accepted: 25 July 2013

Published: 31 July 2013

\section{References}

1. Bucaneve G, Micozzi A, Menichetti F, Martino P, Dionisi MS, Martinelli G, et al: Levofloxacin to prevent bacterial infection in patients with cancer and neutropenia. N Engl J Med 2005, 353:977-987.

2. Cullen M, Steven N, Billingham $L$, Gaunt $C$, Hastings $M$, Simmonds $P$, et al: Antibacterial prophylaxis after chemotherapy for solid tumors and lymphomas. N Engl J Med 2005, 353:988-998.

3. Gafter-Gvili A, Fraser A, Paul M, Leibovici L: Meta-analysis: antibiotic prophylaxis reduces mortality in neutropenic patients. Ann Intern Med 2005, 142:979-995.

4. Reuter S, Kern W, Sigge A, Dohner H, Marre R, Kern P, et al: Impact of fluoroquinolone prophylaxis on reduced infection-related mortality among patients with neutropenia and hematologic malignancies. Clin Infect Dis 2005, 40:1087-1093.

5. Gafter-Gvili A, Paul M, Fraser A, Leibovici L: Effect of quinolone prophylaxis in afebrile neutropenic patients on microbial resistance: systematic review and meta-analysis. J Antimicrob Chemother 2007, 59:5-22.

6. Leibovici L, Paul M, Cullen M, Bucaneve G, Gafter-Gvili A, Fraser A, et al: Antibiotic prophylaxis in neutropenic patients: new evidence, practical decisions. Cancer 2006, 107:1743-1751.

7. Von BH, Sigge A, Bommer M, Kern W, Marre R, Dohner $H$, et al: J Antimicrob Chemother 2006, 58:891-894.

8. Kern W, Steib-Bauert M, De WK, Reuter S, Bertz H, Frank U, et al: Fluoroquinolone consumption and resistance in haematology-oncology patients: ecological analysis in two university hospitals 1999-2002. J Antimicrob Chemother 2005, 55:57-60.

9. Paterson DL, Mulazimoglu L, Casellas JM, Ko WC, Goossens H, Von GA, et al: Epidemiology of ciprofloxacin resistance and its relationship to extended-spectrum beta-lactamase production in Klebsiella pneumoniae isolates causing bacteremia. Clin Infect Dis 2000, 30:473-478.

10. Schwaber MJ, Navon-Venezia S, Schwartz D, Carmeli Y: High levels of antimicrobial coresistance among extended-spectrum-beta-lactamaseproducing Enterobacteriaceae. Antimicrob Agents Chemother 2005, 49:2137-2139.

11. Spanu T, Luzzaro F, Perilli M, Amicosante G, Toniolo A, Fadda G: Occurrence of extended-spectrum beta-lactamases in members of the family Enterobacteriaceae in Italy: implications for resistance to beta-lactams and other antimicrobial drugs. Antimicrob Agents Chemother 2002, 46:196-202.

12. Lautenbach E, Strom BL, Bilker WB, Patel JB, Edelstein PH, Fishman NO: Epidemiological investigation of fluoroquinolone resistance in infections due to extended-spectrum beta-lactamase-producing Escherichia coli and Klebsiella pneumoniae. Clin Infect Dis 2001, 33:1288-1294.

13. Trecarichi EM, Tumbarello M, Spanu T, Caira M, Fianchi L, Chiusolo P, et al: Incidence and clinical impact of extended-spectrum-beta-lactamase (ESBL) production and fluoroquinolone resistance in bloodstream infections caused by Escherichia coli in patients with hematological malignancies. J Infect 2009, 58:299-307.

14. Wener KM, Schechner V, Gold HS, Wright SB, Carmeli Y: Treatment with fluoroquinolones or with beta-lactam-beta-lactamase inhibitor 
combinations is a risk factor for isolation of extended-spectrum-betalactamase-producing Klebsiella species in hospitalized patients. Antimicrob Agents Chemother 2010, 54:2010-2016.

15. Oliveira AL, De SM, Carvalho-Dias VM, Ruiz MA, Silla L, Tanaka PY, et al: Epidemiology of bacteremia and factors associated with multi-drug-resistant gram-negative bacteremia in hematopoietic stem cell transplant recipients. Bone Marrow Transplant 2007, 39:775-781.

16. Gales AC, Castanheira M, Jones RN, Sader HS: Antimicrobial resistance among Gram-negative bacilli isolated from Latin America: results from SENTRY Antimicrobial Surveillance Program (Latin America, 2008-2010). Diagn Microbiol Infect Dis 2012, 73:354-360.

17. From the Immunocompromised Host Society: The design, analysis, and reporting of clinical trials on the empirical antibiotic management of the neutropenic patient. Report of a consensus panel. J Infect Dis 1990, 161:397-401.

18. Performance Standards for Antimicrobial Susceptibility Testing M100 S20. USA: Clinical and Laboratory Standards Institute; 2010.

19. Jarlier V, Nicolas MH, Fournier G, Philippon A: Extended broad-spectrum beta-lactamases conferring transferable resistance to newer beta-lactam agents in Enterobacteriaceae: hospital prevalence and susceptibility patterns. Rev Infect Dis 1988, 10:867-878.

20. Renders N, Romling Y, Verbrugh H, Van BA: Comparative typing of Pseudomonas aeruginosa by random amplification of polymorphic DNA or pulsed-field gel electrophoresis of DNA macrorestriction fragments. J Clin Microbiol 1996, 34:3190-3195.

21. Versalovic J, Koeuth T, Lupski JR: Distribution of repetitive DNA sequences in eubacteria and application to fingerprinting of bacterial genomes. Nucleic Acids Res 1991, 19:6823-6831.

22. Mahenthiralingam E, Campbell ME, Foster J, Lam JS, Speert DP: Random amplified polymorphic DNA typing of Pseudomonas aeruginosa isolates recovered from patients with cystic fibrosis. J Clin Microbiol 1996, 34:1129-1135.

23. Freifeld AG, Bow EJ, Sepkowitz KA, Boeckh MJ, Ito Jl, Mullen CA, et al: Clinical practice guideline for the use of antimicrobial agents in neutropenic patients with cancer: 2010 Update by the Infectious Diseases Society of America. Clin Infect Dis 2011, 52:427-431.

24. Wingard JR, Eldjerou L, Leather $\mathrm{H}$ : Use of antibacterial prophylaxis in patients with chemotherapy-induced neutropenia. Curr Opin Hematol 2012, 19:21-26.

25. Eleutherakis-Papaiakovou E, Kostis E, Migkou M, Christoulas D, Terpos E, Gavriatopoulou M, et al: Prophylactic antibiotics for the prevention of neutropenic fever in patients undergoing autologous stem-cell transplantation: results of a single institution, randomized phase 2 trial. Am J Hematol 2010, 85:863-867.

26. Chong $Y$, Yakushiji H, Ito $Y$, Kamimura T: Clinical impact of fluoroquinolone prophylaxis in neutropenic patients with hematological malignancies. Int J Infect Dis 2011, 15:e277-e281.

27. Craig M, Cumpston AD, Hobbs GR, Devetten MP, Sarwari AR, Ericson SG: The clinical impact of antibacterial prophylaxis and cycling antibiotics for febrile neutropenia in a hematological malignancy and transplantation unit. Bone Marrow Transplant 2007, 39:477-482

28. Gomez L, Garau J, Estrada C, Marquez M, Dalmau D, Xercavins M, et al: Ciprofloxacin prophylaxis in patients with acute leukemia and granulocytopenia in an area with a high prevalence of ciprofloxacin-resistant Escherichia coli. Cancer 2003, 97:419-424.

29. Cremet L, Caroff N, Dauvergne S, Reynaud A, Lepelletier D, Corvec S: Prevalence of plasmid-mediated quinolone resistance determinants in ESBL Enterobacteriaceae clinical isolates over a 1-year period in a French hospital. Pathol Biol (Paris) 2011, 59:151-156.

doi:10.1186/1471-2334-13-356

Cite this article as: Garnica et al: Ciprofloxacin prophylaxis in high risk neutropenic patients: effects on outcomes, antimicrobial therapy and resistance. BMC Infectious Diseases 2013 13:356.

\section{Submit your next manuscript to BioMed Central and take full advantage of:}

- Convenient online submission

- Thorough peer review

- No space constraints or color figure charges

- Immediate publication on acceptance

- Inclusion in PubMed, CAS, Scopus and Google Scholar

- Research which is freely available for redistribution

Submit your manuscript at www.biomedcentral.com/submit
C Biomed Central 